\title{
Rezension:
}

\section{Rechtspolitische Schriften}

Augspurg, Anita: Rechtspolitische Schriften. Kommentierte Studienausgabe. Herausgegeben und kommentiert von Christiane Henke (= Rechtsgeschichte und Geschlechterforschung 16). Köln: Böhlau Verlag Köln 2013. ISBN 978-3-412-21075-5; 423 S.; EUR 69,90.

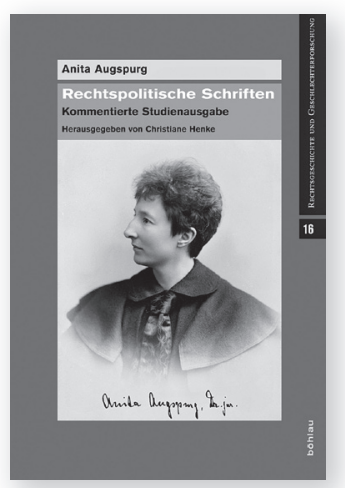

\section{Dr. Marion Röwekamp, Lateinamerika Institut, Freie Universität Berlin}

Über die Feministin und Juristin Anita Augspurg wurde verglichen mit vielen anderen wichtigen Persönlichkeiten der deutschen Frauenbewegung schon viel geforscht und veröffentlicht. Dies liegt nicht nur daran, dass sie eine der federführenden Feministinnen ihrer Zeit und eine der ersten deutschen studierten Juristinnen war, sondern auch, weil sie mit ihrer Lebensgefährtin Lida Gustava Heymann eine Autobiografie hinterlassen hat, ${ }^{1}$ die eine Lebensbeschreibung für Biografen deutlich erleichtert. So hat sie bisher auch einige Biografinnen gefunden, u.a. Christiane Henke, die 1995 eine Monografie über die ersten Juristinnen der deutschen Frauenbewegung, unter ihnen Anita Augspurg, und im Jahr 2000 eine Biografie über Anita Augspurg veröffentlicht hat ${ }^{2}$ und daher als intime Kennerin der Feministin quasi dazu prädestiniert ist, auch ihr Werk herauszugeben.

Christiane Henke hat in ihrem neuen Buch nun aus den über 500 Publikationen, die sie von Anita Augspurg ausfindig machen konnte, 76 repräsentative Schriften ausgewählt und kommentiert, um das Werk der Juristin für die wissenschaftliche Öffentlichkeit leichter zugänglich zu machen. Tatsächlich war es bisher kompliziert, auf Augspurgs Arbeiten zuzugreifen, weil sie über eine Anzahl von Zeitschriften verteilt waren, die nicht überall einsehbar sind. Gleichzeitig waren bisher aber auch nicht alle Schriften bibliografisch bekannt, bereits mit dem Auffindigmachen der Texte, der Sammlung und Zugänglichmachung der umfangreichen Bibliografie der Werke Augspurgs hat sich Christiane Henke der Wissenschaft verdient gemacht. Der Band enthält darüber hinaus eine sehr knappe Begründung der Textauswahl, Textanordnung und Textgestalt, eine auch sehr kurze Biografie Augspurgs, und ein Personen-, Sach- und Ortsregister.

Die Texte selbst hat Henke in drei größere Gruppen eingeteilt und innerhalb dieser Gruppen chronologisch geordnet. Die erste größte Gruppe stellen die „Texte zur Rechtslage der Frau im Deutschen Kaiserreich" dar und unterteilen sich wiederum in „Texte zur Bildungs- und Erwerbsfragen“, in „Texte zu ethischen, politischen, und sozialen Aspekten der Frauenbewegung,“ „Texte zum Eherecht und zum Bürgerlichen Gesetzbuch“, „Texte zu Sittlichkeitsvorstellungen im Strafrecht“, „Texte zum
Vereins- und Versammlungsrecht“ sowie „Friedenspolitischen Texten“. Die nächste Gruppe stellt die „Texte zum politischen Stimmrecht und zu anderen Wahlrechten" dar und dann kommt die Gruppe zu den „Friedenspolitischen Texten“, „Nachrufen“ sowie den Texten zur Bayerischen Revolution und zum Recht der Weimarer Republik.

Um dem Leser eine ,gut lesbare Textfassung“ zu bieten, hat sich Henke gegen den Druck von Faksimiles entschieden sowie gleichzeitig die Originale vorsichtig korrigiert und modernisiert. Als Kennerin wenigstens eines Teils von Augspurgs Texten im Original kann ich versichern, dass das gut gelungen ist und tatsächlich die „individuellen Eigenheiten der ursprünglichen Textgestalt“ (377) nicht zerstört worden ist.

1857 geboren war Anita Augspurg die zweite Juristin Deutschlands und gleichzeitig eine der radikalsten und intellektuellsten Vertreterinnen der deutschen Frauenbewegung. Sie arbeitete als Schauspielerin und Fotografin in München, während sie gleichzeitig frauenpolitisch tätig wurde. Weil sie als Frau in Deutschland nicht zum Studium zugelassen wurde, studierte und promovierte sie Jura in Zürich. Nach ihrer Rückkehr nach Deutschland wurde sie zu einer der zentralsten Figuren der deutschen Frauenbewegung, insbesondere wenn es sich um rechtspolitische Fragen handelte, speziell des Ehe- und des Wahlrechts für Frauen. Bis zum Ende der Weimarer Republik war sie frauenpolitisch aktiv, gab Zeitschriften heraus und zählte während des ersten Weltkriegs gemeinsam mit Lida Gustava Heymann zu den Gründerinnen der Internationalen Frauenliga für Frieden und Freiheit. Nach der Machtübernahme Hitlers 1933 ging Anita Augspurg gemeinsam mit Lida Gustava Heymann ins Exil in die Schweiz, wo sie im Jahr 1943 starb.

Mit ihrem Rechtsstudium in der Schweiz, also gerade über 30 Jahre alt, begann die Veröffentlichungstätigkeit Augspurgs. Ihre Rückkehr nach Deutschland fiel mit einer der lebhaftesten Zeiten der deutschen Frauenbewegung zusammen. Im Zusammenhang mit der anstehenden Kodifikation des Bürgerlichen Gesetzbuchs (BGB) diskutierte die Frauenbewegung erstmals die Rechtsstellung der Frau, hier vor allem im Familien- und dort insbesondere im Eherecht. In diesem Kontext konnte Anita Augspurg ihre neu erworbenen Fachkenntnisse unmittelbar nutzen, tatsächlich war sie es, die 1895 erstmals die Rechtsfrage als Mittelpunkt

1 Lida Gustava Heymann, Erlebtes - Erschautes. Deutsche Frauen kämpfen für Freiheit, Recht und Frieden, hg v. Margrit Twellmann. Meisenheim am Glaan: Anton Hain 1972, (Neuauflage: Königstein: Ulrike Helmer 1992).

2 Siehe z.B.: Ute Gerhard, Anita Augspurg (1857 - 1943): Juristin, Feministin, Pazifistin, in: Streitbare Juristen, hg v. Thomas Blanke et al., Baden-Baden: Nomos 1988, S. 92-103; Christiane Berneike, Die Frauenfrage ist Rechtsfrage. Die Juristinnen der deutschen Frauenbewegung und das Bürgerliche Gesetzbuch, BadenBaden: Nomos 1995; Christiane Henke, Anita Augspurg. Reinbek: Rowohlt 2000; Susanne Kinnebrock, Anita Augspurg (1857-1943). Feministin und Pazifistin zwischen Journalismus und Politik. Eine kommunikationshistorische Biographie. Herbolzheim: Centaurus 2005. 
der Forderungen der Frauenbewegung zu formulieren suchte: „Die Frauenfrage ist zwar zum großen Teil Nahrungsfrage, aber vielleicht in noch höherem Maße Kulturfrage, [...] in allererster Linie ist sie Rechtsfrage, weil nur auf der Grundlage verbürgerter Rechte, nicht idealer [...] an ihre sichere Lösung überhaupt gedacht werden kann.“ (88) Augspurg war der Ansicht, dass ohne die rechtliche Gleichstellung der Frau alle Errungenschaften der Frauenbewegung bloßes Stückwerk bleiben mussten. In dieser Erkenntnis war sie ihrer Zeit weit voraus. So begann sie früher als die anderen Frauenrechtlerinnen die Ausbildung von Juristinnen zu fordern, um eigenes Personal für den Kampf zu haben.

Vor allem stellte sie ihre juristischen Kenntnisse nun aber dem Kampf um die Kodifikation des BGB mit seinen frauenrechtlich diskriminierenden Bestimmungen zur Verfügung. ${ }^{3}$ Sie war die Einzige, die 1905 die grundsätzliche Annahme des Familiennamens des Mannes bereits in Frage stellte (10) und sie war eine der ersten Frauen, die sich zur Reform des Ehegüterrechts äußerte. Das BGB hatte eine Verwaltungsgemeinschaft als gesetzlichen Güterstand bestimmt, nach dem dem Ehemann die Verwaltung und die Nutznießung des von der Ehefrau eingebrachten Vermögens oblag und sie von der Verwaltung ihres eigenen Vermögens ausschloss. Während die Frauenbewegung ihrer Zeit Gütertrennung und den Abschluss von Eheverträgen forderte, um das Vermögen der verheirateten Frauen vor Missbrauch zu schützen und gleichzeitig die eigenen Verfügungsbefugnis zu erhalten, forderte Augspurg 1899 bereits weitergehend auch die Einführung einer Errungenschaftsgemeinschaft, also die finanzielle Beteiligung der Ehefrau an dem während der Ehe erwirtschafteten Vermögens (125). Später wurde diese Forderung ähnlich von den Juristinnen der Weimarer Republik wieder aufgegriffen und als Rechtsforderungen der Deutschen Frauenbewegung einheitlich vom Parlament und dem Reichsjustizministerium eingefordert und schließlich 1958 gesetzlich umgesetzt.

Auspurgs Reden, Vorträge, Artikel und Aufsätze zur Rechtslage der Frau zeigen, wie früh sie schon umfassende Rechtsreformen im Familienrecht forderte, die so radikal und juristisch anspruchsvoll waren, dass es noch bis 1977 dauerte, bis sie zum Teil etwas abgeändert in der Bundesrepublik umgesetzt wurden. So forderte sie auch früh das Wahlrecht für Frauen, als hinsichtlich dessen Forderung die Frauenbewegung noch gespalten war. Ähnlich wie nur die progressivsten Juristen ihrer Zeit forderte Augspurg die Interpretation der Weimarer Reichsverfassung als bindendes Recht und kritisierte den fehlenden Individualrechtsschutz (284). Daneben beobachtete sie kritisch die Gleichstellung der Frau in allen anderen gesetzlichen Texten. Über 40 Jahre kämpfte sie für gleiche Rechte von Frauen und wurde derart, wie Stephan Meder, der Herausgeber der Reihe, es in seiner Einleitung formuliert „als juristische Vordenkerin der organisierten Frauen ihrer Zeit [...] zur maßgeblichen Weggbereiterin “ (9) für gleiche Rechte für Frauen im Kaiserreich und der Weimarer Republik.

Es ist anzunehmen, dass Augspurgs andere Texte ähnlich radikal und progressiv sind, aber mir fehlt die fachliche Kompetenz, dies zu beurteilen. Das wiederum macht einen Schwachpunkt der Edition deutlich. Nach der Lektüre sollte der Leser grundsätzlich in der Lage sein, das Schaffen Augspurgs kontextualisieren zu können. Zwar bieten die sehr guten Kommentierungen der Herausgeberin eine Verortung der konkreten Texte, aber das genügt nicht, um das Potential des Denkens und Schaffens Augspurgs in Relation verstehen zu können. Nicht nur hätten die Texte innerhalb Augspurgs Leben kontextualisiert werden müssen, sondern vor allem aber auch innerhalb des Kontexts ihrer Zeit. Dies nicht nur innerhalb der Frauenbewegung, um den „radikalen“ Inhalt ihrer Forderungen selbst innerhalb einer als frauenpolitisch fortschrittlich denkenden Gruppe begreifen zu können, sondern auch die juristischen Texte innerhalb der juristischen Vergleichstexte, die Friedenstexte innerhalb deren Feld etc. Dazu wäre ideal gewesen, wenn die Herausgeberin den drei großen Textgruppen (Texte zur Rechtslage der Frau im Deutschen Kaiserreich, die friedenspolitischen Texte sowie den Texte zur politischen Lage) eine Einführung vorangestellt hätte oder zumindest eine längere Einleitung geschrieben hätte. So erfährt der Leser nur aus dem kurzen Vorwort Stephan Meders, dass Anita Augspurgs Werk seiner Zeit weit voraus war.

Für den wissenschaftlich Arbeitenden wären an manchen Stellen auch umfangreichere Kommentierungen nötig gewesen, zumal die oben angesprochene grundsätzliche Kontextualisierung des Schaffens Augspurgs fehlt. Insbesondere fehlen an den meisten Stellen weitergehende Verweise bzw. die Nachweise der Kommentare, was den Leser oft ratlos zurücklässt, würde er an manchen Stellen gerne den interessanten Kommentierungen nachgehen und vertiefend arbeiten. Dies schränkt die wissenschaftliche Nutzung der Studienausgabe leider ein.

So bedauernswert diese Einschränkungen sind, ist der Quellenband trotzdem für einschlägige rechtshistorische und historische Forschungen ein Glücksfall und unverzichtbar für jeden, der sich mit der bürgerlichen Frauenbewegung im Kaiserreich und der Weimarer Republik beschäftigt. Jeder, der bisher versucht hat, in dieser Zeit zu forschen, weiß, wie kompliziert und ungeheuer mühsam es ist, auch nur einen groben Überblick über das Schaffen einer Persönlichkeit zu erhalten, selbst wenn sie rechtshistorisch unumstritten von Bedeutung ist wie die Anita Augspurgs. Wenn dann ein Teil des Werks auch noch lesbar aufbereitet und gut kommentiert ist, kann dem Herausgeber nicht genug gedankt werden. Christiane Henke hat mit der Veröffentlichung des Werks von Augspurg nicht nur das Schaffen einer der interessantesten Persönlichkeiten der deutschen, radikalen Frauenbewegung für die Öffentlichkeit zugänglich gemacht, sondern auch der Forschung einen großen Dienst erwiesen.

3 Siehe zu diesem Thema z.B. Christiane Berneike, Die Frauenfrage ist Rechtsfrage. Die Juristinnen der deutschen Frauenbewegung und das Bürgerliche Gesetzbuch, Baden-Baden: Nomos 1995; Arne Duncker, Gleichheit und Ungleichheit in der Ehe. Persönliche Stellung von Frau und Mann im Recht der ehelichen Lebensgemeinschaft 1700-1914, Köln: Böhlau 2003; Andrea Czelk, „Privilegierung“ und Vorurteil. Positionen der Bürgerlichen Frauenbewegung zum Unehelichenrecht und zur Kindstötung im Kaiserreich, Köln: Böhlau 2005; Jens Lehmann, Die Ehefrau und ihr Vermögen. Reformforderungen der bürgerlichen Frauenbewegung zum Ehegüterrecht um 1900, Köln: Böhlau 2006; Tanja-Carina Riedel, Gleiches Recht für Frau und Mann. Die bürgerliche Frauenbewegung und die Entstehung des BGB, Köln: Böhlau 2008. 